\title{
Electronic and geometric structure of graphene/SiC(0001) decoupled by lithium intercalation
}

\author{
F. Bisti, ${ }^{1,}$ G. Profeta, ${ }^{1,2}$ H. Vita, ${ }^{3}$ M. Donarelli, ${ }^{1}$ F. Perrozzi, ${ }^{1}$ P. M. Sheverdyaeva, ${ }^{4}$ P. Moras, ${ }^{4}$ K. Horn, ${ }^{3}$ and L. Ottaviano ${ }^{1,2}$ \\ ${ }^{1}$ Dipartimento di Scienze Fisiche e Chimiche, Università dell'Aquila, Via Vetoio 10, 67100, L'Aquila, Italy \\ ${ }^{2}$ CNR-SPIN L'Aquila, Via Vetoio 10, 67100 L'Aquila, Italy \\ ${ }^{3}$ Fritz-Haber Institut der Max-Planck Gesellschaft, Department of Physical Chemistry, 14195 Berlin, Germany \\ ${ }^{4}$ Istituto di Struttura della Materia, Consiglio Nazionale delle Ricerche, I-34149 Trieste, Italy \\ (Received 23 October 2014; revised manuscript received 19 May 2015; published 11 June 2015)
}

\begin{abstract}
Graphene formation on top of $\mathrm{SiC}(0001)$ by decoupling the carbon buffer layer through lithium intercalation is investigated. Low-energy electron diffraction and core-level photoemission spectroscopy results show that graphene formation already occurs at room temperature, and that the interface morphology is improved after thermal annealing. Angle-resolved photoemission spectroscopy (ARPES) shows that the resulting graphene layer is strongly $n$-type doped, and in spite of the decoupling by lithium intercalation, a persistent interaction with the substrate imposes a superperiodicity on the graphene band structure that modulates the $\pi$ band intensity and gives rise to quasi- $(2 \times 2) \pi$ replica bands. Through a comparison of the ARPES-derived band structure with density-functional-theory calculations, we assign the observed bands to SiC-derived states and interface-related ones; this assignment permits us to establish that the intercalated lithium occupies the T4 site on the topmost $\mathrm{SiC}$ layer.
\end{abstract}

DOI: 10.1103/PhysRevB.91.245411

PACS number(s): 68.65.Pq, 73.22.Pr, 73.20.At, 71.15.Mb

\section{INTRODUCTION}

The interaction between graphene and adsorbed or intercalated alkali-metal atoms has received considerable attention in recent years, not only because of obvious analogies to the wellknown graphite intercalation compounds [1], but also because the interaction may be used to induce a considerable "chemical doping" [2], and deposition with subsequent intercalation can bring this doping to extreme values [3]. Apart from doping effects, the intercalation process is also an interesting method for the decoupling of graphene from substrates, as in the case of the $\mathrm{SiC}(0001)$ surface with the so-called buffer layer on top, corresponding to the $(6 \sqrt{3} \times 6 \sqrt{3}) R 30^{\circ}$ reconstruction. The intercalation process is able to transform the buffer layer into monolayer graphene by decoupling it from the silicon carbide substrate. Various atomic species such as $\mathrm{H} \mathrm{[4],} \mathrm{Li}$ [5-7], $\mathrm{Au}$ [8], Si [9], O [10], F [11,12], Na [13], and Rb and Cs [14] have been used for this purpose. These intercalated atoms, by saturating the Si dangling bonds, break the buffer layer bonds with the substrate. This process transforms the buffer layer into graphene, which can be quasi-free-standing in the case of $\mathrm{H}$ [4] and $\mathrm{Au}$ [8] or weakly coupled with the intercalates through electron or hole doping effects $[11,13,14]$.

In the present work, we study lithium intercalation in between the carbon buffer layer and the silicon-terminated face of silicon carbide, i.e., $\mathrm{SiC}(0001)$, since the interaction of graphene with lithium attracts special attention for fundamental and technological reasons, related to the fabrication of Li-based batteries [15], possible piezoelectric effects [16], and a predicted superconducting phase [17]. Li deposition on graphene on the $\mathrm{SiC}(0001)$ surface has already been investigated in the case in which an already formed graphene layer is on top of the buffer layer. This results in lithium intercalation in between the substrate and the buffer layer,

\footnotetext{
*Present address: Paul Scherrer Institut, Swiss Light Source, CH-5232 Villigen PSI, Switzerland; federico.bisti@psi.ch
}

converting it into bilayer graphene [5-7]. On the other hand, the interaction of lithium with $\mathrm{SiC}(0001)$ just in the presence of the buffer layer has only been investigated by lowenergy electron microscopy and $\mathrm{x}$-ray photoemission electron microscopy [5]. Furthermore, the intercalation process of the $\mathrm{Li}$ atoms has been theoretically modeled considering different absorption sites [18,19].

Here we report on a comprehensive investigation on the latter system, performed by means of complementary lowenergy electron diffraction (LEED) and synchrotron radiation core-level spectroscopy, before and after $\mathrm{Li}$ intercalation. We demonstrate that the buffer layer decoupling is obtained directly at room temperature, and a successive annealing procedure improves the intercalation quality, reducing the excess of lithium on top. The experiments were complemented by angle-resolved photoemission spectroscopy (ARPES) measurements on the intercalated system, showing a strong $n$-type doped graphene layer and a strongly changed band structure near the Fermi level. By means of tight-binding calculations, we demonstrate that the interaction between the graphene layer and the substrate gives rise to a complex double-folded $\pi$-band structure. Finally, first-principles density-functional-theory (DFT) calculations allow us to recognize spectral features in the ARPES measurements that enable us to identify the $\mathrm{Li}$ intercalation site.

\section{EXPERIMENT}

We used a nitrogen-doped $\left[n \approx(1-2) \times 10^{18} \mathrm{~cm}^{-3}\right] 6 \mathrm{H}-$ $\mathrm{SiC}(0001)$ wafer, terminated by $\mathrm{Si}$ atoms in the topmost layer. The wafer was etched in molecular hydrogen at high temperature in order to remove polishing damage. The annealing temperature and time in an argon atmosphere were optimized to obtain the $(6 \sqrt{3} \times 6 \sqrt{3}) R 30^{\circ}$ reconstruction, in line with the results in Ref. [20]. The samples were then transferred in air to the photoemission setup and cleaned in situ by annealing at $400^{\circ} \mathrm{C}$. LEED, ARPES, and core-level photoemission spectroscopy experiments were performed at 
the VUV-Photoemission beamline (Elettra Trieste) in ultrahigh vacuum (UHV) with a base pressure of $4 \times 10^{-10}$ mbar. $\mathrm{Li}$ was deposited on the sample from an alkali-metal getter source (SAES Getters) while keeping the sample at room temperature. For the core-level spectra, the beamline and the spectrometer parameters were adjusted to achieve an overall spectral resolution of $70 \mathrm{meV}$ at room temperature. The energy and angular resolution of the ARPES data were $25 \mathrm{meV}$ and $0.3^{\circ}$, respectively, using a photon energy of $65 \mathrm{eV}$. All spectra were referenced to the Fermi level of graphene. The core-level spectra were decomposed using a linear combination of Voigt functions with a Shirley background. The particular shape of the graphene core-level component was obtained by fitting the data reported in Ref. [21] for the case of alkaline metals, using two Doniach-Sunjic functions. The obtained component was then adapted for our present spectra, by only an adjustment of binding energy position and intensity, without changing its shape. Momentum distribution curve (MDC) and energy distribution curve (EDC) analyses were used in the ARPES data for detecting the dispersion of different bands. In the first case, the curves were fitted using a linear combination of Lorentzian functions with the addition of a second-order polynomial for the background. In the second case, a linear combination of a Gaussian function and first-order polynomial was used. The graphene $(13 \times 13)$ supercell electronic structure was obtained by a tight-binding model in the first-nearest-neighbor approximation (1NN TB), considering only $p_{z}$ orbitals [22]. DFT calculations were performed using the Vienna Ab-initio Simulation Package (VASP) [23] with a plane-wave cutoff of $350 \mathrm{eV}$ and $12 \times 12 \times 1$ Monkhorst-Pack grid sampling for the charge-density integration. The PBE exchange-correlation functional was used.

\section{RESULTS AND DISCUSSION}

The structural evolution of the system under investigation was monitored by LEED. In Fig. 1(a), we show the LEED pattern of the ex situ prepared $\mathrm{SiC}$ surface terminated by the buffer layer, which is typical for the $(6 \sqrt{3} \times 6 \sqrt{3}) R 30^{\circ}$ reconstruction [20,24]. Figures 1(b) and 1(c) show the LEED patterns of the same surface after $\mathrm{Li}$ deposition at room temperature [panel (b)] and upon further annealing (10 min) at $360{ }^{\circ} \mathrm{C}$ [panel (c)]. The two LEED patterns in (b) and (c) are practically identical apart from an observable sharpening of the LEED spots in (c). Both diagrams [(b) and (c)] exhibit the hexagonal patterns of the SiC [labeled "SiC $(1 \times 1)$ ") in Fig. 1(c)] and graphene $1 \times 1$ [labeled "Gr $(1 \times 1)$ "] phases, while other extra spots around the graphene ones can be barely distinguished. This is already clear evidence of the buffer layer decoupling process induced by exposure to lithium. Furthermore, the remaining extra spots, around the "Gr $(1 \times 1)$ " ones, are interpreted as a periodic "corrugation" of the graphene layer [labeled as "Gr $(13 \times 13)$ "], induced by the remaining coupling with the substrate.

The C $1 s, \mathrm{Si} 2 p$, and $\mathrm{Li} 1 s$ core-level spectra corresponding to the LEED patterns of Fig. 1 are reported in Fig. 2 (top, middle, and bottom panels, respectively). Overall, we have followed the spectra decomposition proposed in Ref. [6], apart from using a doublet for the description of the graphene component. This particular component shape has been reported (a)

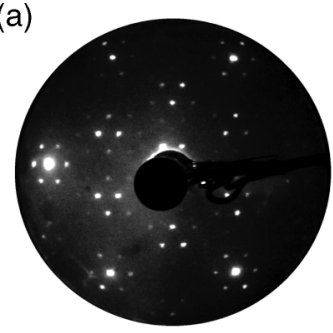

(b)

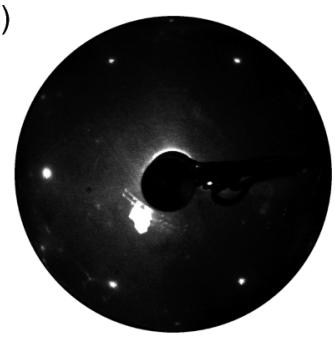

(c)

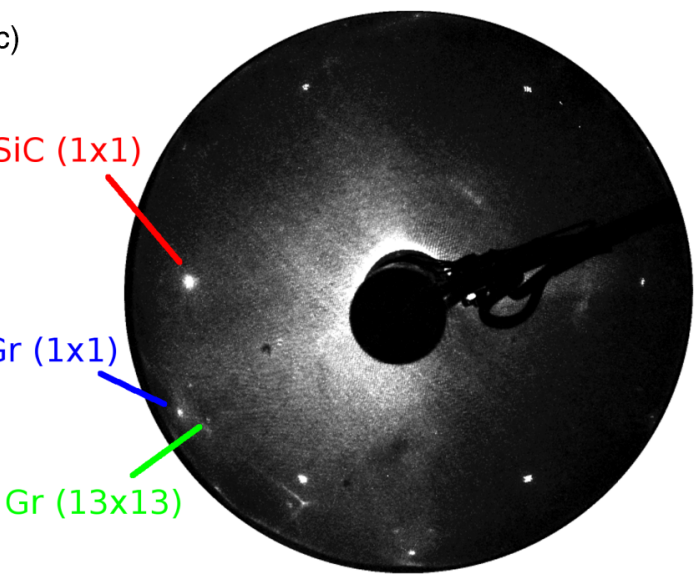

FIG. 1. (Color online) LEED patterns at $100 \mathrm{eV}$ of pristine $\mathrm{SiC}$ (a), after Li deposition (b) and after annealing in $\mathrm{UHV}$ at $360{ }^{\circ} \mathrm{C}$ (c).

in the case of quasi-free-standing graphene doped by alkaline metals (as Li) [21], and we demonstrate that it agrees with our case. Starting from the $(6 \sqrt{3} \times 6 \sqrt{3}) R 30^{\circ}$ reconstructed surface (red curve in Fig. 2, top panel), we decompose its C $1 s$ spectrum with two components, one coming from the bulk (labeled as "SiC") and the other one from the buffer layer ("buff"). The corresponding Si $2 p$ spectrum (red curve in Fig. 2, middle panel) shows just a doublet coming from the bulk SiC substrate ("SiC") [6]. After Li deposition, the buffer layer component of the $\mathrm{C} 1 s$ spectrum (blue curve in the top panel of Fig. 2) is mainly transformed into the doublet component (of Ref. [21]) assigned to the formation of graphene (referred to as "Gr"). A broad and unstructured component at the highest-binding-energy side is instead assigned to photoelectrons from $\mathrm{C}$ atoms near structural defects (labeled "def" in Fig. 2). On the other hand, in the Si $2 p$ spectrum (middle panel of Fig. 2), a new component ("Li-Si") is assigned to the topmost $\mathrm{Si}$ atoms that were previously bonded to the buffer layer $\mathrm{C}$ atoms and are now effectively decoupled and interact with the intercalated Li. The intensity of this component is slightly reduced after annealing, pointing to a partial desorption of the intercalated Li atoms. A third minor component, assigned again to $\mathrm{Si}$ atoms surrounding structural defects (referred to as "def" in Fig. 2), is required to provide a good fit to the data. In terms of binding-energy positions, the "SiC" components (in the C $1 s$ and $\mathrm{S} 2 p$ spectra) are observed to rigidly shift by $1.8 \mathrm{eV}$ to lower binding energies with respect to the corresponding components before Li deposition, and by $1.2 \mathrm{eV}$ for the annealed Li exposed-surfaces. Such rigid shifts have already been observed and attributed to the formation of a dipole layer at the interface between graphene and $\mathrm{SiC}$, due 

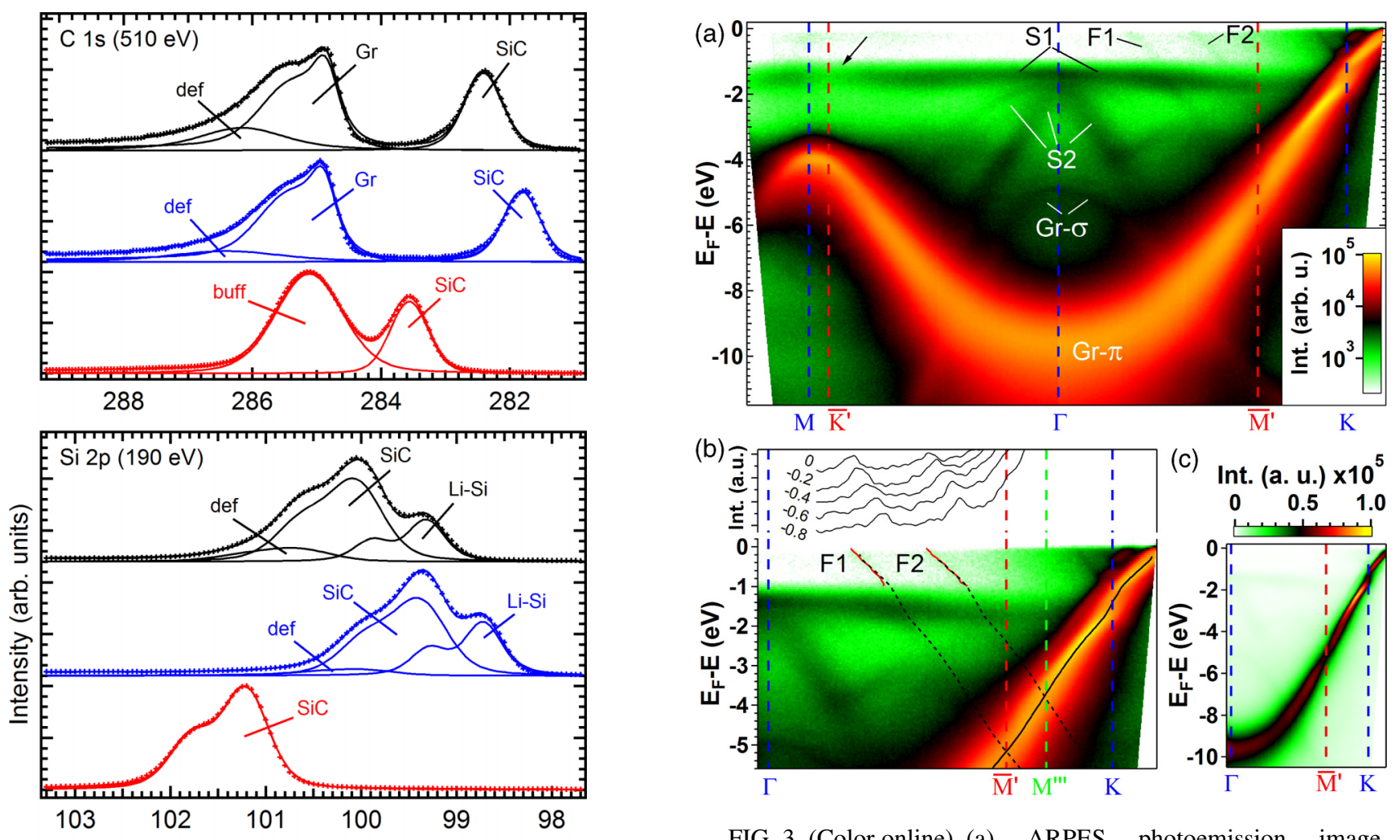

FIG. 3. (Color online) (a) ARPES photoemission image, recorded at a photon energy of $65 \mathrm{eV}$, after Li deposition and thermal annealing along the $M-\Gamma$ and $\Gamma-K$ directions of graphene. Data reported using a logarithmic color scale. (b) Bottom left, enlargement of the ARPES data near the "F1" and "F2" features. The black line indicates the graphene $\pi$-band and the red lines the "F1" and "F2" features. The dotted black lines, continuing the "F1" and "F2" features, are obtained by reflection of the black line through the $\bar{M}^{\prime}$ and $M^{\prime \prime \prime}$ vertical lines. Inset: MDCs at different binding energies, integrated over $( \pm 0.1 \mathrm{eV})$ and smoothed with a binomial procedure. (c) ARPES image along $\Gamma-K$ direction of graphene using a linear color scale.

FIG. 2. (Color online) C $1 s$ (top), Si $2 p$ (middle), and Li $1 s$ (bottom) core-level spectra of pristine $\mathrm{SiC}$ (red), after room-temperature $\mathrm{Li}$ deposition (blue) and after $360^{\circ} \mathrm{C}$ annealing (black).

to the presence of intercalated Li [6]. The further reduction of this energy shift after annealing is consistent with partial desorption of the intercalated $\mathrm{Li}$, as observed by the intensity reduction of the "Li-Si" component.

The Li $1 s$ core-level line shape (bottom panel in Fig. 2) after deposition (blue curve in the bottom panel of Fig. 2) exhibits a dominant component centered at $54.7 \mathrm{eV}$, accompanied by weaker structures observed at the higher-binding-energy side. Very likely, excess $\mathrm{Li}$ atoms form a thin metallic film that carries plasmonic losses that smear out the other features observed in the Li $1 s$ core level. After annealing of the surface, we observe an overall energy shift of $0.6 \mathrm{eV}$ to higher binding energies fully consistent with the rigid shifts in the corresponding $\mathrm{C} 1 s$ and $\mathrm{Si} 2 p$ spectra. Furthermore, from a quantitative comparison of the Li $1 s$ to Si $2 p$ spectra, we estimate that $60 \%$ of the deposited lithium is effectively desorbed. The Li $1 s$ spectrum becomes more clearly structured into three components: (i) the component at the lower binding

energy is assigned to $\mathrm{Li}$ adsorbed on top of graphene (" $\mathrm{Li}$ top"); (ii) the component observed at intermediate energy is assigned to intercalated $\mathrm{Li}$ atoms effectively saturating the Si dangling bonds of the substrate ("Li-Si"); (iii) finally, the component at the high-binding-energy side is assigned to $\mathrm{Li}$ atoms neighboring structural defects ("def").

Both core-level spectroscopy and LEED clearly indicate that $\mathrm{Li}$ is effective in decoupling the buffer layer, with the formation of monolayer graphene already upon roomtemperature deposition. However, the successive thermal treatment allows the lithium excess to be removed and improves the long-range surface morphology, as observed by LEED. For the above reasons, the ARPES analysis presented in the following focuses on data from samples annealed after $\mathrm{Li}$ deposition.

The ARPES measurements are depicted in Fig. 3, along the $M-\Gamma$ and $\Gamma-K$ directions of the graphene $(1 \times 1)$ Brillouin zone (in blue) corresponding to the $\bar{K}^{\prime}-\Gamma$ and $\Gamma-\bar{M}^{\prime}$ directions of the $\mathrm{SiC}(1 \times 1)$ Brillouin zone (in red). The most intense signal of the ARPES data comes from the graphene $\pi$-band ("Gr- $\pi$ "), which is one order of magnitude more intense than 
the other ARPES features [25]. The Dirac point energy is around $-1.4 \mathrm{eV}$, indicating a strong $n$-type chemical doping of the detached graphene layer [3]. Comparing this result with the data of McChesney et al. [3], who performed intercalation and adsorption of calcium to induce extremely strong $n$-type doping in order to create an extended Van Hove singularity in the vicinity of the Fermi level, we note that the present level of doping is hardly enough to induce a superconductivity scenario. The graphene $\sigma$-band, with a reduced spectral intensity, can be found at $-5 \mathrm{eV}$ at the $\Gamma$ point ("Gr- $\sigma$ ") and disperses downward in energy. Other spectral features at lower binding energy, clearly visible along both directions, are a slightly downward-dispersing band ("S1") and three more strongly downward-dispersing ones ("S2") all originating at about $-1.4 \mathrm{eV}$ at the $\Gamma$ point. These features correspond to states of $\mathrm{Li}$ and the topmost layer of the $\mathrm{SiC}$ substrate, as we will demonstrate by comparison with DFT calculations below. An indication for this assignment can already be found in the "S1" intensity reduction near the $\mathrm{SiC}(1 \times 1) \bar{K}^{\prime}$ point (as indicated by the arrow), so this band is consistent with the $\mathrm{SiC}(1 \times 1)$ periodicity. Finally, two other weak bands labeled "F1" and "F2" are observed, and they are analyzed further in Fig. 3(b) where MDCs (obtained by energy integration around $\pm 0.1 \mathrm{eV}$ of the center value shown next to the curves) are reported in order to better distinguish those features from the background. At the bottom of the panel, the results of a complete MDC analysis are superimposed over the ARPES image. The black line is obtained from the centers of the Lorentzian functions fitting the peaks of the $\pi$-band dispersion, and the red lines reproduce the "F1" and "F2" dispersions from a similar MDC analysis. The black dotted lines are results of the analysis of the $\pi$-band folded back by the two symmetry points $\left(\bar{M}^{\prime}\right.$ and $\left.M^{\prime \prime \prime}\right)$. The overlap between the red and black dotted lines, i.e., the linear shape and the gradient of the bands, suggests that these two features correspond to a folding of the graphene $\pi$-band around two particular points along the $\Gamma-K$ direction: $\bar{M}^{\prime}$ of the $\mathrm{SiC}(1 \times 1)$ mesh, and $M^{\prime \prime \prime}$, according to the graphene $(13 \times 13)$ periodicity. Around the $\bar{M}^{\prime}$ point of the $\mathrm{SiC}(1 \times 1)$, we note an intensity reduction of the graphene $\pi$-band [better visible in Fig. 3(c) with a linear color scale], pointing to an influence of the substrate periodicity on the graphene band structure.

Within a simple tight-binding model, it is possible to demonstrate that these two replicas are folded bands of the graphene $(13 \times 13)$ periodicity observed in LEED; they are also the closest ones to approximate the $(2 \times 2)$ periodicity. In Fig. 4, we show the Brillouin zones for the different graphene $[(1 \times 1),(2 \times 2)$, and $(13 \times 13)]$ and $\operatorname{SiC}(1 \times 1)$ periodicities. In the same panel, below the Brillouin zones, the horizontal lines show the relevant high symmetry points for the different periodicities along the $\Gamma-K$ direction of the graphene $(1 \times 1)$ mesh. In Fig. 4(b), we show the results of a tight-binding calculation of monolayer graphene, considering the different unit cells. In the manifold of bands arising from the $(13 \times 13)$ mesh (green lines), two bands marked as "F1" and "F2" (red lines) are found, backfolded at the $\bar{M}^{\prime}$ (or $\left.\Gamma^{\prime \prime \prime}\right)$ and $M^{\prime \prime \prime}$ points, which are exactly on the right and left side of the backfolded $\pi$-band (in black) of the $(2 \times 2)$ periodicity. We also notice that their Dirac points are exactly at $5 / 13$ and $8 / 13$ along the $\Gamma-K$ segment. Therefore, they are analogous to the quasi $(2 \times 2)$ (a)
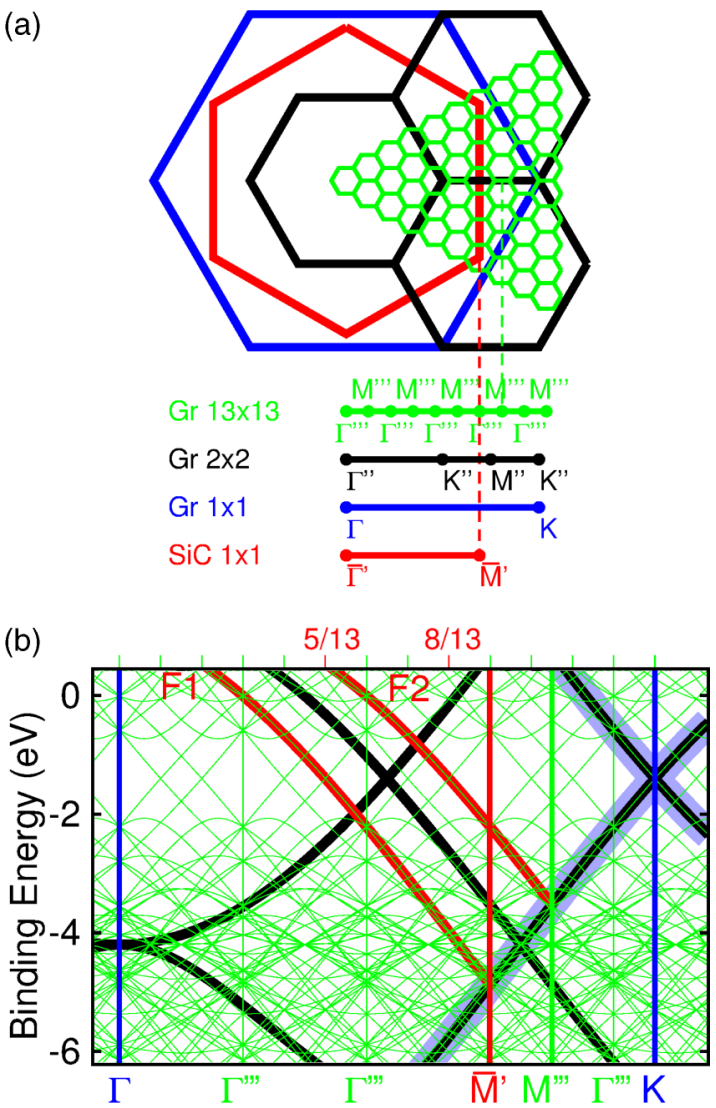

FIG. 4. (Color online) (a) Top: 2D Brillouin zone of $\mathrm{SiC}(1 \times 1)$ (red hexagon) and graphene $(1 \times 1),(2 \times 2)$, and $(13 \times 13)$ (blue, black, and green hexagons); bottom: principal symmetrical points for the different periodicities along the $\Gamma-K$ direction of graphene $(1 \times 1)$. (b) Tight-binding band structures of graphene $(1 \times 1)$ unit cell (light blue curves), along the $\Gamma-K$ directions, folded onto the $(2 \times 2)($ black$)$ and $(13 \times 13)($ green $)$ ones.

replicas observed in the graphene layer on top of the SiC buffer layer [26]. The origin of these features has been explained as a consequence of a strong $(2 \times 2)$ charge modulation induced by the substrate [27]. A similar backfolding of the $\pi$-band was also observed for epitaxial graphene on iridium [28], and the signal intensity of the folded bands has been related to graphene corrugation induced by the substrate [29]. Therefore, our system is another interesting example of the modification of the electronic structure of graphene induced by a different substrate periodicity even though the interaction between $\mathrm{SiC}$ and the graphene layer is strongly suppressed by the presence of the intercalated lithium atoms.

As a final part of this work, we demonstrate that the "S1" and "S2" features are related to states of the lithiumdoped SiC substrate, and that the shape and location of the "S1" band contain information on the Li intercalation site. For this purpose, we have performed DFT calculations considering a graphene layer on the Si-terminated surface of $\mathrm{SiC}$, intercalated with $\mathrm{Li}$ atoms. The system periodicity corresponds to the $(6 \sqrt{3} \times 6 \sqrt{3}) R 30^{\circ}$ supercell of $\mathrm{SiC}$, which is commensurate with the $(13 \times 13)$ supercell of graphene [19]. Since the calculation of this supercell requires an enormous amount of computational time, we have adopted 
the strategy taken by Mattausch et al. and Pankratov et al. $[30,31]$ to calculate the structural and electronic properties of a reduced $\mathrm{SiC}(\sqrt{3} \times \sqrt{3}) R 30^{\circ}$ supercell with four $\mathrm{SiC}$ bilayers and $10 \AA$ of vacuum, with a subsequent unfolding procedure to permit a direct comparison with the experimental data. On this unit cell, it is possible to accommodate a $2 \times 2$ graphene supercell, stretched by $8.4 \%$. We have studied the interaction between lithium and graphene and $\mathrm{SiC}$ considering one $\mathrm{Li}$ atom per $\mathrm{SiC}(1 \times 1)$ unit cell. To find the most favorable adsorption site on the SiC surface, we calculated the total energy of the system, placing lithium on three different, symmetry-inequivalent sites, namely the onefold-coordinated site on top of surface $\mathrm{Si}$ atoms [T1, see Fig. 5(a)], the threefold-coordinated hollow site of the $\mathrm{SiC}(0001)$ surface [H3, see Fig. 5(b)], and the fourfold-coordinated site on top of the $\mathrm{C}$ subsurface layer [T4, see Fig. 5(c)]. The DFT predicts, in this supercell, that T4 is the most stable site, the H3 site has a higher total energy of $0.24 \mathrm{eV}$ per Li atom, and the $\mathrm{T} 1$ site is $0.55 \mathrm{eV}$ per Li atom [32]; clearly, there are large differences that are well above the confidence interval for such total energy calculations. Before comparing them with the ARPES data, the calculated band structures need to be unfolded from the supercell (utilized in the calculations) onto the unit cell of graphene and $\mathrm{SiC}$. Therefore, for each calculated eigenvalue, we associate a weight proportional to the projected component of the related eigenfunction, at the supercell wave vector, onto those at the wave vector of the unit cell [33]. Finally, the bands are assigned to $\mathrm{Li}$ (green), $\mathrm{Si}$ (red), and $\mathrm{C}$ states (blue). After this unfolding procedure, the band structures are reported in Figs. 5(a), 5(b), and 5(c) for the T1, H3, and T4 intercalation sites, respectively.

The prediction of a slightly dispersing band around $-0.4 \mathrm{eV}$ binding energy, which we assign to the "S1" feature observed in the experimental data, is of central importance for the present calculations. The projection onto the atomic state assigns this band as originating from the interaction between the intercalated $\mathrm{Li}$ atoms and the $\mathrm{SiC}$ topmost layer. For each intercalation site, we have a different energy dispersion: upward-dispersing for $\mathrm{T} 1$, almost nondispersing for $\mathrm{H} 3$, and downward-dispersing for T4. An excellent agreement with the experimental data is obtained for the dispersion of the $\mathrm{T} 4$ site, as shown in Fig. 5(c), where the centers of the Gaussian function fitting the experimental "S1" band dispersion (dark gray line) are reported. The experimental data were shifted to a lower binding energy of $1.22 \mathrm{eV}$. This difference between experimental and theoretical data is due to several factors, such as remaining $\mathrm{Li}$ atoms on top of graphene that were not accounted in the theoretical model, and the increased graphene work function induced by its stretched lattice [34]. To overcome these problems, in Fig. 5(d) we report the calculated band structure for the Li-T4 system (without any further structural relaxation) with an additional doping induced by adding one electron per $(6 \sqrt{3} \times 6 \sqrt{3}) R 30^{\circ}$ supercell, compensated by a uniform positive background. The main obtained effect is a downward shift to lower energy for all the bands, which is more evident for the $\mathrm{SiC}$ bands than for the graphene ones. The "S1" feature is now at $-1.6 \mathrm{eV}$ and the graphene Dirac point at $-1.3 \mathrm{eV}$, much closer to the experimental values $(-1.4 \mathrm{eV}$ for both cases). Moreover, by comparing data in Figs. 3 and 5, we assign the "S2"
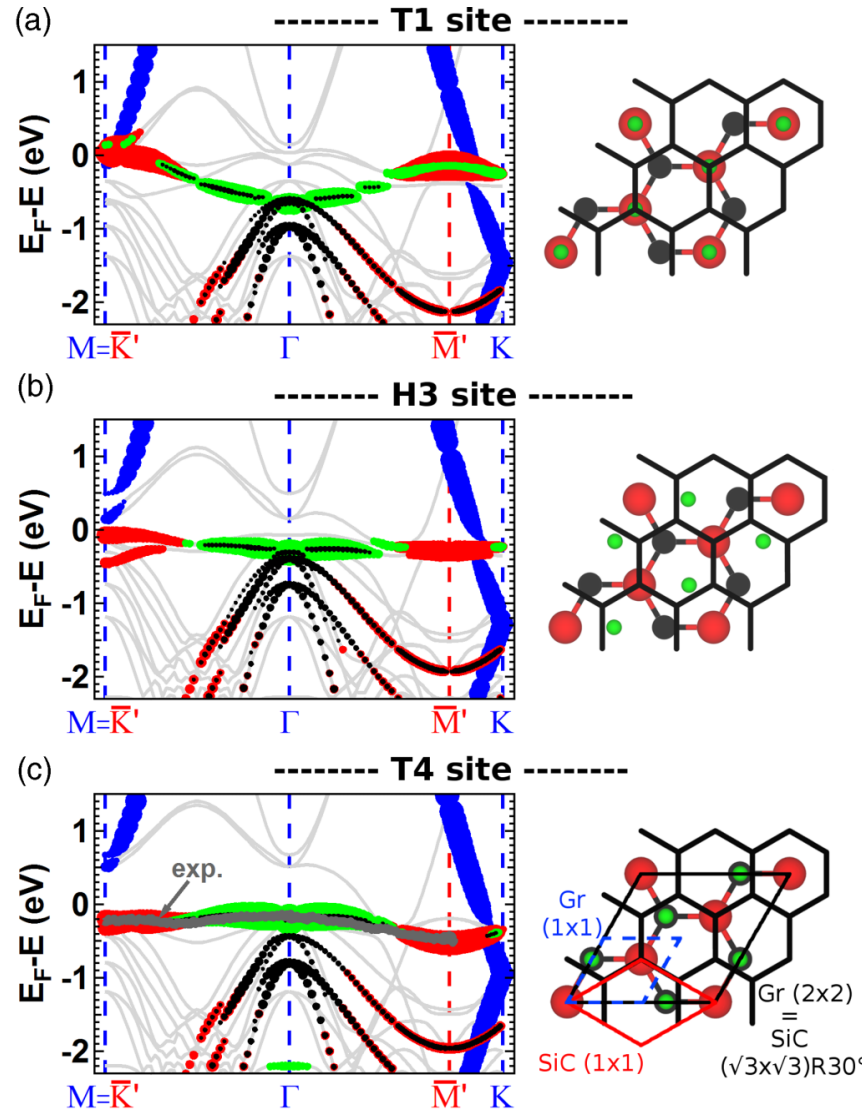

(d) T4 site, additional doping

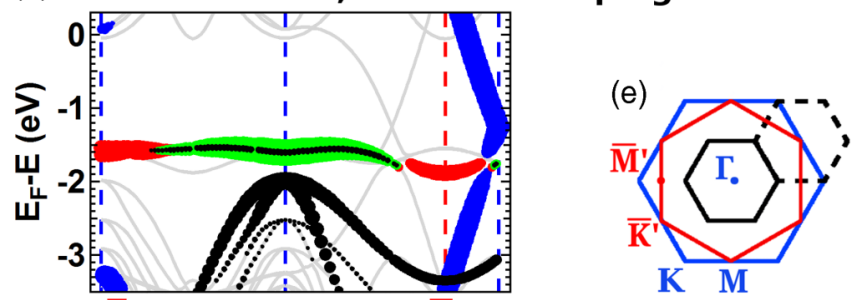

FIG. 5. (Color online) DFT band structures, along the $M-\Gamma$ and $\Gamma-K$ graphene direction, for the system after Li intercalation on the $\mathrm{T} 1$ (a), H3 (b), and T4 (c) sites, and on the T4 site with additional doping introduced (d). The band structures are weighted by the projecting factor on three different states bases: atomic Li states in $\mathrm{SiC}(1 \times 1)$ (green dots), atomic Si states in $\mathrm{SiC}(1 \times 1)$ (red dots), and atomic $\mathrm{C}$ states in $\mathrm{SiC}(1 \times 1)$ and in graphene $(1 \times 1)$ (black and blue dots, respectively, with weight values divided by 2 for clarity). The dark gray line in (c) represents the experimental data of the "S1" feature shifted by $1.22 \mathrm{eV}$ to lower binding energy. Next to the band structures, structural models of the system upon Li intercalation are shown. Parallelograms in the T4 sketch are the unit cells of graphene $(1 \times 1)$ (blue), of $\mathrm{SiC}(1 \times 1)$ (red), and the $(2 \times 2)$ supercell of graphene or $\mathrm{SiC}(\sqrt{3} \times \sqrt{3}) R 30^{\circ}$ (black). (e) $2 \mathrm{D}$ Brillouin zone of graphene $(1 \times 1)$, of $\mathrm{SiC}(1 \times 1)$ and graphene $(2 \times 2)$, or $\operatorname{SiC}(\sqrt{3} \times$ $\sqrt{3}) R 30^{\circ}$ (blue, red, and black hexagons, respectively).

feature to bands of the $\mathrm{SiC}$ carbon atoms of the topmost layer. Therefore, the good agreement obtained with the experimental data supports the $\mathrm{T} 4$ site as the favorite intercalation site for the Li. 


\section{CONCLUSION}

We have investigated the $(6 \sqrt{3} \times 6 \sqrt{3}) R 30^{\circ}$ buffer layer on $\mathrm{SiC}$ (0001), and its decoupling from the substrate through $\mathrm{Li}$ deposition and intercalation by means of LEED and core-level photoemission spectroscopy. Our data confirm that $\mathrm{Li}$ intercalates between $\mathrm{SiC}$ and detaches the buffer layer to produce a monolayer of graphene, which is strongly $n$-type doped. By analyzing faint features in the ARPES data and assigning them to backfolding through the difference in the meshes involved, we demonstrate that the detached graphene layer still interacts with the Li-SiC substrate strongly enough to give rise to two backfolded bands in the electronic structure. Finally, we compare the experimental band structure observed by ARPES with our DFT calculations; by unfolding the calculated band structure from the $(\sqrt{3} \times \sqrt{3}) R 30^{\circ}$ supercell onto the $(1 \times 1)$ unit cell of graphene and $\mathrm{SiC}$, we find good agreement between the experimental and calculated bands, based on the occupancy of lithium on the T4 site in intercalated graphene.

\section{ACKNOWLEDGMENTS}

We thank Th. Seyller and his group for supplying the buffer layer sample. F.B. acknowledges Sincrotrone Trieste S.C.p.A. for financial support. This work was supported by the EuroGRAPHENE program through project SpinGraph (DFG project HO 797/18-1), the Deutsche Forschungsgemeinschaft through Schwerpunktprogramm 1459 "Graphene" (project DE 1679/3-1), the FP7 European project SUPER-IRON (Grant Agreement No. 283204), the Italian Ministry of University Research through the PRIN 2012 project, and a CINECA award under the ISCRA initiative for the availability of high performance computing resources and support.
[1] T. Enoki, M. Suzuki, and M. Endo, Graphite Intercalation Compounds and Applications (Oxford University Press, New York, 2003).

[2] T. Ohta, A. Bostwick, T. Seyller, K. Horn, and E. Rotenberg, Science 313, 951 (2006).

[3] J. L. McChesney, A. Bostwick, T. Ohta, T. Seyller, K. Horn, J. González, and E. Rotenberg, Phys. Rev. Lett. 104, 136803 (2010).

[4] C. Riedl, C. Coletti, T. Iwasaki, A. A. Zakharov, and U. Starke, Phys. Rev. Lett. 103, 246804 (2009).

[5] C. Virojanadara, A. A. Zakharov, S. Watcharinyanon, R. Yakimova, and L. I. Johansson, New J. Phys. 12, 125015 (2010)

[6] C. Virojanadara, S. Watcharinyanon, A. A. Zakharov, and L. I. Johansson, Phys. Rev. B 82, 205402 (2010).

[7] K. Sugawara, K. Kanetani, T. Sato, and T. Takahashi, AIP Adv. 1, 022103 (2011).

[8] I. Gierz, T. Suzuki, R. T. Weitz, D. S. Lee, B. Krauss, C. Riedl, U. Starke, H. Höchst, J. H. Smet, C. R. Ast, and K. Kern, Phys. Rev. B 81, 235408 (2010).

[9] C. Xia, S. Watcharinyanon, A. A. Zakharov, R. Yakimova, L. Hultman, L. I. Johansson, and C. Virojanadara, Phys. Rev. B 85, 045418 (2012).

[10] M. H. Oliveira, Jr., T. Schumann, F. Fromm, R. Koch, M. Ostler, M. Ramsteiner, T. Seyller, J. M. J. Lopes, and H. Riechert, Carbon 52, 83 (2013).

[11] A. L. Walter, K.-J. Jeon, A. Bostwick, F. Speck, M. Ostler, T. Seyller, L. Moreschini, Y. S. Kim, Y. J. Chang, K. Horn, and E. Rotenberg, Appl. Phys. Lett. 98, 184102 (2011).

[12] S. L. Wong, H. Huang, Y. Wang, L. Cao, D. Qi, I. Santoso, W. Chen, and A. T. S. Wee, ACS Nano 5, 7662 (2011).

[13] S. Watcharinyanon, L. I. Johansson, C. Xia, and C. Virojanadara, J. Appl. Phys. 111, 083711 (2012).

[14] S. Watcharinyanon, C. Virojanadara, and L. Johansson, Surf. Sci. 605, 1918 (2011).

[15] E. Yoo, J. Kim, E. Hosono, H.-s. Zhou, T. Kudo, and I. Honma, Nano Lett. 8, 2277 (2008).

[16] M. T. Ong and E. J. Reed, ACS Nano 6, 1387 (2012).

[17] G. Profeta, M. Calandra, and F. Mauri, Nat. Phys. 8, 131 (2012).
[18] Y. Li, G. Zhou, J. Li, J. Wu, B.-L. Gu, and W. Duan, J. Phys. Chem. C 115, 23992 (2011).

[19] I. Deretzis and A. LaMagna, Phys. Rev. B 84, 235426 (2011).

[20] K. V. Emtsev, F. Speck, T. Seyller, L. Ley, and J. D. Riley, Phys, Rev. B 77, 155303 (2008).

[21] A. V. Fedorov, N. I. Verbitskiy, D. Haberer, C. Struzzi, L. Petaccia, D. Usachov, O. Y. Vilkov, D. V. Vyalikh, J. Fink, M. Knupfer, B. Büchner, and A. Grüneis, Nat. Commun. 5, 3257 (2014).

[22] R. Saito, G. Dresselhaus, and M. S. Dresselhaus, Physical Properties of Carbon Nanotubes (Imperial College Press, London, 1998).

[23] G. Kresse and J. Furthmüller, Phys. Rev. B 54, 11169 (1996).

[24] K. Emtsev, A. Bostwick, K. Horn, J. Jobst, G. Kellogg, L. Ley, J. McChesney, T. Ohta, S. Reshanov, J. Rhrl, E. Rotenberg, A. Schmid, D. Waldmann, H. Weber, and T. Seyller, Nat. Mater. 8, 203 (2009).

[25] A second $\pi$-band parallel to the main one is also barely visible. Having a lower binding energy, we interpreted it as coming from small initial graphene coverage present before the $\mathrm{Li}$ deposition. As reported in Ref. [20], samples prepared to induce the $(6 \sqrt{3} \times 6 \sqrt{3}) R 30^{\circ}$ reconstruction are usually characterized by the coexistence of seed graphene patches on top of the buffer layer. In our case, a graphene-related component in the $\mathrm{C} 1 \mathrm{~s}$ spectrum could not be unequivocally disentangled, therefore, by comparing our C $1 s$ spectra with those reported in Ref. [20], such initial graphene coverage has to be lower than 0.3 ML. Because the ARPES intensity of these features is one order of magnitude lower than that of the main feature, we can roughly estimate the initial graphene coverage be $0.1 \mathrm{ML}$.

[26] K. Nakatsuji, Y. Shibata, R. Niikura, F. Komori, K. Morita, and S. Tanaka, Phys. Rev. B 82, 045428 (2010).

[27] F. Varchon, P. Mallet, J.-Y. Veuillen, and L. Magaud, Phys. Rev. B 77, 235412 (2008).

[28] E. Starodub, A. Bostwick, L. Moreschini, S. Nie, F. E. Gabaly, K. F. McCarty, and E. Rotenberg, Phys. Rev. B 83, 125428 (2011). 
[29] D. Usachov, A. Fedorov, O. Vilkov, V. K. Adamchuk, L. V. Yashina, L. Bondarenko, A. A. Saranin, A. Grüneis, and D. V. Vyalikh, Phys. Rev. B 86, 155151 (2012).

[30] A. Mattausch and O. Pankratov, Phys. Rev. Lett. 99, 076802 (2007).

[31] O. Pankratov, S. Hensel, P. Götzfried, and M. Bockstedte, Phys. Rev. B 86, 155432 (2012).
[32] We have also performed the same calculations without the graphene layer on $\mathrm{SiC}$. T4 remains the most stable adsorption site, and the $\mathrm{H} 3$ (T1) site has a higher total energy of $0.33 \mathrm{eV}$ $(0.90 \mathrm{eV})$ per Li atom.

[33] G. Profeta et al. (unpublished).

[34] S.-M. Choi, S.-H. Jhi, and Y.-W. Son, Phys. Rev. B 81, 081407 (2010). 